\title{
CONVOLUTION OPERATORS ON LEBESGUE SPACES OF THE HALF-LINE
}

\author{
BY \\ VICTOR W. DANIEL( $\left.{ }^{1}\right)$
}

\begin{abstract}
In this paper we determine the lattice of closed invariant subspaces for certain convolution operators on Lebesgue spaces $L^{p}(d \sigma)$ where $\sigma$ is a suitable weighted measure on the half-line. We exploit the rather close relationship between convolution operators and the collection of right translation operators $\left\{T_{\lambda}\right\}_{\lambda \geqq 0}$ on $L^{p}(d \sigma)$. We show that a convolution operator $K$ and the collection $\left\{T_{\lambda}\right\}_{\lambda \geq 0}$ have the same lattice of closed invariant subspaces provided the kernel $k$ of $K$ is a cyclic vector. The converse also holds if we assume in addition that the closed span of $\left\{T_{\lambda} k\right\}_{\lambda \geqq 0}$ is all of $L^{p}(d \sigma)$. We show that the lattice of closed right translation invariant subspaces of $L^{p}(d \sigma)$ is totally ordered by set inclusion whenever $\sigma$ has compact support. Thus in this case a convolution operator $K$ is unicellular if and only if its kernel is a cyclic vector. Finally, we show for suitable weighted measures $\sigma$ on the half-line that the convolution operators on $L^{p}(d \sigma)$ are Volterra.
\end{abstract}

1. Introduction. In this paper we study convolution operators on Lebesgue spaces $L^{p}(d \sigma), 1 \leqq p<\infty$, where $\sigma$ is a measure on $[0, \infty)$ of the form $d \sigma=\alpha d x$ where $\alpha \geqq 0$ is a nonincreasing function on $(0, \infty)$. A convolution operator $K$ on $L^{p}(d \sigma)$ is an operator of the form

$$
K g=\int_{0}^{x} k(x-t) g(t) d t
$$

where the kernel $k$ is a Lebesgue measurable function on $(0, \infty)$. Under suitable integrability conditions on $k$ we show that $K$ is everywhere defined and bounded on $L^{p}(d \sigma)$ and furthermore

$$
K \in \text { strongly closed algebra generated by }\left\{T_{\lambda}\right\}_{\lambda \geqq 0}
$$

where $\left\{T_{\lambda}\right\}_{\lambda \geqq 0}$ is the collection of right translation operators on $L^{p}(d \sigma)$.

In general, the lattice of closed invariant subspaces of $K$ is not known. However, from above we see that any closed right translation invariant subspace of $L^{p}(d \sigma)$ is also invariant under $K$. In this paper we determine sufficient conditions on the kernel $k$ so that these subspaces represent all the closed invariant subspaces of $K$.

Received by the editors February 8, 1971 and, in revised form, June 3, 1971.

AMS 1970 subject classifications. Primary 47G05; Secondary 44A35, 45D05.

Key words and phrases. Convolution operator, right translation operator, strongly closed algebra, invariant subspace, Bochner integral, Volterra, quasinilpotent, unicellular, cyclic vector.

(1) A generalization of part of the results of the author's doctoral dissertation written under the guidance of James Rovnyak at the University of Virginia.

Copyright (C) 1972, American Mathematical Society 
Under additional hypotheses on $\sigma$ we get a simple necessary and sufficient condition on $k$; namely that $k$ be a cyclic vector for $K$.

Of special interest is the case where the closed support of $\sigma$ is compact. Then we can show that the collection of closed right translation invariant subspaces of $L^{p}(d \sigma)$ is totally ordered by set inclusion. In this case we determine sufficient conditions on the kernel $k$ so that the convolution operator $K$ is unicellular.

Another problem we investigate is determining conditions on the measure $\sigma$ so that the convolution operators on $L^{p}(d \sigma)$ are Volterra. It is a well-known fact that convolution operators on $L^{p}(0,1)$ are Volterra. So not surprisingly we find that convolution operators on $L^{p}(d \sigma)$ are Volterra whenever $\sigma$ has compact support. For general measures $\sigma$ on the half-line there may exist convolution operators that are not Volterra. For example, there are no nonzero compact convolution operators on either $L^{p}(0, \infty)$ or $L^{p}\left(e^{-x} d x\right)$. However, if $\sigma$ is a suitable weighted measure on the half-line (e.g. $d \sigma=\exp \left(-x^{2}\right) d x$ ), then convolution operators on $L^{p}(d \sigma)$ are Volterra.

2. General development. Throughout this paper $\sigma$ will denote a measure on $[0, \infty)$ of the form $d \sigma=\alpha d x$ where $\alpha \geqq 0$ is a nonincreasing function on $(0, \infty)$. We will add additional hypotheses on $\sigma$ as the need arises. Unless otherwise stated, $p$ will denote any real number satisfying $1 \leqq p<\infty$ and $q$ will denote the conjugate to $p$ given by $1 / p+1 / q=1$.

We find it convenient to regard functions in $L^{p}(d \sigma)$ as being defined on the whole real line and having the value 0 on $(-\infty, 0)$. With this convention we define the right translation operators $\left\{T_{\lambda}\right\}_{\lambda \geqq 0}$ on $L^{p}(d \sigma)$ by $\left(T_{\lambda} g\right) x=g(x-\lambda)$ for all $g$ in $L^{p}(d \sigma)$. Clearly $\left\|T_{\lambda}\right\| \leqq 1$ since $\alpha$ is nonincreasing on $(0, \infty)$. In fact, $\left\{T_{\lambda}\right\}_{\lambda \geqq 0}$ is a strongly continuous semigroup of operators on $L^{p}(d \sigma)$.

Given two Lebesgue measurable functions $k$ and $g$ on $(0, \infty)$ we define the convolution $k * g$ in the usual manner by

$$
(k * g) x=\int_{0}^{x} k(x-t) g(t) d t
$$

at each $x$ for which the Lebesgue integral exists.

Suppose $k$ is a Lebesgue measurable function on $(0, \infty)$ and let $K$ be the convolution operator on $L^{p}(d \sigma)$ with kernel $k$ defined by $K g=k * g$. We prefer to view the convolution operator $K$ in terms of Bochner integrals as follows:

$$
\begin{aligned}
K g & =\int_{0}^{x} g(x-t) k(t) d t=\int_{0}^{x}\left(T_{t} g\right) x k(t) d t=\left[\int_{0}^{\infty} T_{t} g k(t) d t\right](x) \\
& =\int_{[0, \infty)} T_{t} g d \mu(t) \quad \text { (Bochner } \mu \text {-integral) }
\end{aligned}
$$

where $\mu$ is the measure on $[0, \infty)$ defined by $d \mu=k d t$. The Bochner integral need not exist in general without additional assumptions on $\mu$ and $g$. If the Bochner 
integral does exist, then by a theorem of S. Bochner (see Yosida [5]) we must have

$$
\int_{[0, \infty)}\left\|T_{t} g\right\|_{p}|d \mu(t)|<\infty
$$

Fortunately this condition is strong enough to guarantee the existence of the Bochner integral when $\mu$ is a Baire measure on $[0, \infty)$. We state without proof the following lemmas:

LEMMA 1. Suppose $\mu$ is a complex Baire measure on $[0, \infty)$ and set

$$
D(K)=\left\{f \in L^{p}(d \sigma): \int_{[0, \infty)}\left\|T_{t} f\right\|_{p}|d \mu(t)|<\infty\right\} .
$$

Then we can define a convolution operator $K$ on $D(K)$ by $K f=\int_{(0, \infty)} T_{t} f d \mu(t)$ where the Bochner $\mu$-integral exists for all $f$ in $D(K)$. Furthermore,

$$
K f=\lim _{n \rightarrow \infty} \sum_{j=1}^{n^{2}}\left(T_{j / n} f\right) \mu([(j-1) / n, j / n))
$$

for all $f$ in $D(K)$. Thus if $D(K)=L^{p}(d \sigma)$ it follows that $K$ is bounded and

$K \in$ strongly closed algebra generated by $\left\{T_{\lambda}\right\}_{\lambda \geqq 0}$.

LEMMA 2. Suppose $\mu$ is a complex Baire measure on $[0, \infty)$ and $K$ is the convolution operator on $D(K)$ defined in Lemma 1 . Then for each $f$ in $D(K)$ the function $t \rightarrow\left(T_{t} f\right) x$ $=f(x-t)$ is in $L^{1}(d \mu)$ for $\sigma$-a.e. $x$ and furthermore

$$
(K f) x=\int_{[0, \infty)} f(x-t) d \mu(t) \text { for } \sigma \text {-a.e. } x .
$$

LEMMA 3. For each positive integer $n$, set

$$
\begin{aligned}
& \delta_{n}(x)=n \quad \text { if } 1 / n \leqq x \leqq 2 / n, \\
& =0 \text { otherwise. }
\end{aligned}
$$

Then $\left\{\delta_{n}\right\}_{n=1}^{\infty}$ is an approximate identity for convolution on $L^{p}(d \sigma)$. That is, $\lim \delta_{n} * g$ $=g$ for all $g$ in $L^{p}(d \sigma)$ where the limit is taken in the norm of $L^{p}(d \sigma)$.

Although Lemmas 1 and 2 are stated for convolution operators $K$ on $L^{p}(d \sigma)$ whose kernels are Baire measures, we are primarily interested in the case where $\mu$ is a Baire measure on $[0, \infty)$ of the form $d \mu=k d t$. Then $k$ is the usual kernel function for $K$ and

$$
K g=\int_{0}^{\infty}\left(T_{t} g\right) k(t) d t=k * g
$$

for all $g$ in $D(K)$. Note that $g \in D(K)$ implies that $(k * g) x$ exists for $\sigma$-a.e. $x$ and $k * g \in L^{p}(d \sigma)$.

Of special interest is the case where the kernel $k \in L^{1}\left(\left\|T_{t}\right\| d t\right)\left(\left\|T_{t}\right\|\right.$ denotes the norm of $T_{t}$ acting on $\left.L^{p}(d \sigma)\right)$. Then by Lemma $1, K$ is everywhere defined on $L^{p}(d \sigma)$ and $\|K\| \leqq\|k\|$ where $\|k\|$ denotes the $L^{1}\left(\left\|T_{t}\right\| d t\right)$ norm of $k$. One easily computes 
that $K^{n} g=\left(k^{* n}\right) * g$ where $k^{* n}=k * k * \cdots * k$ ( $n$ times). Each $k^{* n} \in L^{1}\left(\left\|T_{\imath}\right\| d t\right)$ since $L^{1}\left(\left\|T_{t}\right\| d t\right)$ is closed under the binary operation $*$ (this follows from the estimate $\left\|T_{t+s}\right\| \leqq\left\|T_{t}\right\|\left\|T_{s}\right\|$, valid for all $s, t \geqq 0$ ).

Now we can state the main result of this section.

THEOREM 1. Suppose $k \in L^{1}\left(\left\|T_{t}\right\| d t\right)$ and $K$ is the convolution operator on $L^{p}(d \sigma)$ with kernel $k$. Assume $\left\{k^{* n}\right\}_{n=1}^{\infty}$ is fundamental in $L^{1}\left(\left\|T_{t}\right\| d t\right)$. Then

strongly closed algebra generated by $K$

$$
=\text { strongly closed algebra generated by }\left\{T_{\lambda}\right\}_{\lambda \geqq 0} .
$$

Proof. By Lemma 1 we already have $K \in$ strongly closed algebra generated by $\left\{T_{\lambda}\right\}_{\lambda \geqq 0}$. Conversely, we intend to show that each $T_{\lambda} \in$ strongly closed algebra generated by $K$.

Fix $\lambda \geqq 0$ and let $\left\{\delta_{n}\right\}_{n=1}^{\infty}$ be the approximate identity of Lemma 3. For each positive integer $n$ choose $p_{n} \in$ linear $\operatorname{span}\left\{k^{* n}\right\}_{n=1}^{\infty}$ so that $\left\|p_{n}-T_{\lambda} \delta_{n}\right\| \leqq 1 / n$. Let $p_{n}(K)$ be the polynomial in $K$ defined by $p_{n}(K) g=p_{n} * g$. Then for any $g$ in $L^{p}(d \sigma)$ we have

$$
\begin{aligned}
\left\|p_{n}(K) g-T_{\lambda} g\right\|_{p} & \leqq\left\|p_{n} * g-T_{\lambda} \delta_{n} * g\right\|_{p}+\left\|T_{\lambda} \delta_{n} * g-T_{\lambda} g\right\|_{p} \\
& \leqq\left\|p_{n}-T_{\lambda} \delta_{n}\right\|\|g\|_{p}+\left\|\delta_{n} * T_{\lambda} g-T_{\lambda} g\right\|_{p} .
\end{aligned}
$$

Thus $\lim p_{n}(K) g=T_{\lambda} g$ for all $g$ in $L^{p}(d \sigma)$. Hence $T_{\lambda} \in$ strongly closed algebra generated by $K$, proving the theorem.

COROLlaRY. Suppose $\alpha$ is submultiplicative (i.e. $\alpha(x+y) \leqq \alpha(x) \alpha(y)$ for all $x, y>0)$ and $k \in L^{1}(d \sigma)$ satisfies

$$
\text { closed span of }\left\{T_{\lambda} k\right\}_{\lambda \geqq 0}=L^{1}(d \sigma) .
$$

Let $K$ be the convolution operator on $L^{1}(d \sigma)$ with kernel $k$. Then the following are equivalent:

(i) $k$ is a cyclic vector for $K$,

(ii) strongly closed algebra generated by $K=$ strongly closed algebra generated by $\left\{T_{\lambda}\right\}_{\lambda \geq 0}$,

(iii) $K$ and $\left\{T_{\lambda}\right\}_{\lambda \geqq 0}$ have the same collection of closed invariant subspaces.

Proof. (i) $\Rightarrow$ (ii). Suppose $k$ is a cyclic vector for $K$. Then $\left\{K^{n} k\right\}_{n=0}^{\infty}=\left\{k^{* n}\right\}_{n=1}^{\infty}$ is fundamental in $L^{1}(d \sigma)$. A short calculation shows that $\left\{k^{* n}\right\}_{n=1}^{\infty}$ is fundamental in $L^{1}\left(\left\|T_{t}\right\| d t\right)$. Now (ii) follows from Theorem 1 .

(ii) $\Rightarrow$ (iii). Obvious.

(iii) $\Rightarrow$ (i). Assume (iii) holds and set $M=$ closed span of $\left\{K^{n} k\right\}_{n=0}^{\infty}$ in $L^{1}(d \sigma)$. Clearly $M$ is a closed invariant subspace for $K$. Then by assumption, $M$ is a closed invariant subspace for $\left\{T_{\lambda}\right\}_{\lambda \geqq 0}$. Using $\left(^{*}\right)$ we get

$$
L^{1}(d \sigma)=\text { closed } \operatorname{span}\left\{T_{\lambda} k\right\}_{\lambda \geqq 0} \subseteq M \subseteq L^{1}(d \sigma) .
$$

Thus $M=L^{1}(d \sigma)$ and so $k$ is a cyclic vector for $K$. This proves (i) and completes the proof of the corollary. 
3. Measures $\sigma$ with compact support. In this section we assume that the closed support of $\sigma$ is compact. Without loss of generality we may assume that supp $\sigma$ $=[0,1]$. Thus $\sigma$ is a measure on $[0,1]$ of the form $d \sigma=\alpha d x$ where $\alpha>0$ is a nonincreasing function on $(0,1)$.

Our development of convolution operators indicates the importance of determining the lattice of closed right translation invariant subspaces of $L^{p}(d \sigma)$. This lattice forms a chain under set inclusion when the measure $\sigma$ has compact support. This result was first proved by S. Agmon [1] for the Lebesgue measure case. The proof for the general case is not difficult using the Titchmarsh convolution theorem. Thus we state without proof the following lemma:

Lemma 4. Suppose $\operatorname{supp} \sigma=[0,1]$. Then the lattice of closed right translation invariant subspaces of $L^{p}(d \sigma)$ is the chain $\left\{M_{a}\right\}_{0 \leqq a \leqq 1}$ where

$$
M_{a}=\left\{f \in L^{p}(d \sigma): f=0 \text { a.e. on }[0, a]\right\} .
$$

An operator is said to be unicellular if its collection of closed invariant subspaces forms a chain under set inclusion. Combining Theorem 1 and Lemma 4 we obtain the following sufficient condition for a convolution operator to be unicellular:

Theorem 2. Suppose supp $\sigma=[0,1]$ and $k \in L^{1}\left(\left\|T_{t}\right\| d t\right)$. Assume $\left\{k^{* n}\right\}_{n=1}^{\infty}$ is fundamental in $L^{1}\left(\left\|T_{t}\right\| d t\right)$. Then the convolution operator $K$ on $L^{p}(d \sigma)$ with kernel $k$ is unicellular, its chain of closed invariant subspaces is $\left\{M_{a}\right\}_{0} \leqq 1$.

So far we have assumed only that $\operatorname{supp} \sigma=[0,1]$. If we assume in addition that $\alpha$ is submultiplicative (i.e. $\alpha(x+y) \leqq \alpha(x) \alpha(y)$ for $x, y>0$ ), then we get the following necessary and sufficient conditions for a convolution operator on $L^{p}(d \sigma)$ to be unicellular:

THEOREM 3. Suppose $\alpha$ is submultiplicative and supp $\sigma=[0,1]$. Suppose $k \in L^{p}(d \sigma)$ and $K$ is the convolution operator on $L^{p}(d \sigma)$ with kernel $k$. Then the following are equivalent:

(i) $K$ is unicellular,

(ii) $k$ is a cyclic vector for $K$,

(iii) strongly closed algebra generated by $K=$ strongly closed algebra generated by $\left\{T_{\lambda}\right\}_{\lambda \geqq 0}$.

Proof. A short calculation shows that $\left\|T_{t}\right\| \leqq \alpha(t)^{1 / p}$. Hence $k \in L^{p}(d \sigma) \subseteq$ $L^{1}\left(\left\|T_{t}\right\| d t\right)$ and so $K$ is everywhere defined and bounded on $L^{p}(d \sigma)$ by Lemma 1 .

(i) $\Rightarrow$ (ii). Suppose $K$ is unicellular. By the Titchmarsh convolution theorem we have $K g=0$ iff $g=0$ a.e. on $\left[0,1-l_{k}\right]$ where

$$
l_{k}=\sup \{l \geqq 0: k=0 \text { a.e. on }[0, l]\} \text {. }
$$

But $\operatorname{dim}(\operatorname{ker} K) \leqq 1$ since $K$ is unicellular. Thus $l_{k}=0$. Set $M=$ closed span of $\left\{K^{n} k\right\}_{n=0}^{\infty}$ in $L^{p}(d \sigma)$. Clearly $M$ is a closed invariant subspace for $K$. Thus $M=M_{a}$ for some $a, 0 \leqq a \leqq 1$. Since $k \in M$ and $l_{k}=0$, it follows that $a=0$. Hence $M=L^{p}(d \sigma)$, proving that $k$ is a cyclic vector for $K$. 
(ii) $\Rightarrow$ (iii). Assume $k$ is a cyclic vector for $K$. Then $\left\{k^{* n}\right\}_{n=1}^{\infty}$ is fundamental in $L^{p}(d \sigma)$, hence fundamental in $L^{1}\left(\left\|T_{t}\right\| d t\right)$. Now (iii) follows from Theorem 1.

(iii) $\Rightarrow$ (i). This follows immediately from Lemma 4 . The proof of the theorem is complete.

An operator is Volterra if it is compact and quasinilpotent (its spectrum contains only the point 0 ). We remark that a convolution operator $K$ on $L^{p}(d \sigma)$ whose kernel $k \in L^{1}\left(\left\|T_{t}\right\| d t\right)$ is Volterra whenever the support of $\sigma$ is compact. The proof involves approximating $K$ in operator norm with convolution operators $K_{n}$ whose kernels $k_{n}$ are continuous on $[0,1]$ and vanish in some neighborhood of 0 . Showing that such convolution operators $K_{n}$ are Volterra is routine.

4. Weighted measures on the half-line. In this section we study convolution operators on Lebesgue spaces $L^{p}(d \sigma)$ where $\sigma$ is a special type of weighted measure on $[0, \infty]$; throughout this section we shall assume $\sigma$ satisfies the following property:

Property P. $\sigma$ is a measure on $[0, \infty)$ of the form $d \sigma=\alpha d x$ where $\alpha \geqq 0$ is a nonincreasing function on $(0, \infty)$ and $\alpha(x+y) \leqq \alpha(x) \alpha(y) \beta(y)^{x}$ for all $x, y>0$. The function $\beta$ on $(0, \infty)$ satisfies

(i) $0 \leqq \beta(t) \leqq 1$ for all $t>0$,

(ii) $C_{p}=\left[\int_{0}^{\infty} \beta(t)^{q / p} d t\right]^{1 / q}<\infty$.

Under these hypotheses we can extend the corollary to Theorem 1 to such $L^{p}(d \sigma)$ spaces when $1 \leqq p<\infty$. Note that for the $p=1$ case that any measure $\sigma$ on $[0, \infty)$ of the form $d \sigma=\alpha d x$ where $\alpha \geqq 0$ is nonincreasing and submultiplicative satisfies Property $\mathbf{P}$ (simply take $\beta=1$ ). Thus the corollary to Theorem 1 will be a special case of our extension. We also can show that convolution operators on $L^{p}(d \sigma)$ are Volterra whenever $\sigma$ satisfies Property $\mathrm{P}$ and $\beta(t)<1$ for all $t>0$. Before proving these results we need the following lemma:

Lemma 5. Suppose $\sigma$ satisfies Property $\mathrm{P}$ and let $k, g \in L^{p}(d \sigma)$. Then $(k * g) x$ exists for $\sigma$-a.e. $x, k * g \in L^{p}(d \sigma)$, and $\|k * g\|_{p} \leqq\left(1+C_{p}\right)\|k\|_{p}\|g\|_{p}$. The convolution operator $K$ on $L^{p}(d \sigma)$ with kernel $k$ is everywhere defined and bounded, and furthermore $K \in$ strongly closed algebra generated by $\left\{T_{\lambda}\right\}_{\lambda \geqq 0}$.

Proof. Set $k_{1}=\chi_{[0,1]} k$ and $k_{2}=\chi_{[1, \infty)} k$ where $\chi_{E}$ denotes the characteristic function of a set $E$. Consider the two Bochner integrals:

$$
\int_{0}^{1}\left(T_{t} g\right) k_{1}(t) d t \text { and } \int_{0}^{\infty}\left(T_{t} k_{2}\right) g(t) d t
$$

Both integrals exist since by Property $\mathrm{P}$ we have the following estimates:

$$
\begin{aligned}
& \int_{0}^{1}\left\|T_{t} g\right\|_{p}\left|k_{1}(t)\right| d t \leqq\left\|k_{1}\right\|_{p}\|g\|_{p}, \\
& \int_{0}^{\infty}\left\|T_{t} k_{2}\right\|_{p}|g(t)| d t \leqq C_{p}\left\|k_{2}\right\|_{p}\|g\|_{p} .
\end{aligned}
$$


By Lemma 2 each Bochner integral can be evaluated for $\sigma$-a.e. $x$ as regular Lebesgue integrals:

$$
\begin{aligned}
& {\left[\int_{0}^{1}\left(T_{t} g\right) k_{1}(t) d t\right] x=\int_{0}^{1} g(x-t) k_{1}(t) d t=\left(k_{1} * g\right) x,} \\
& {\left[\int_{0}^{\infty}\left(T_{t} k_{2}\right) g(t) d t\right] x=\int_{0}^{\infty} k_{2}(x-t) g(t) d t=\left(k_{2} * g\right) x .}
\end{aligned}
$$

Thus $(k * g) x=\left(k_{1} * g\right) x+\left(k_{2} * g\right) x$ exists for $\sigma$-a.e. $x$ and furthermore

$$
\|k * g\|_{p} \leqq\left\|k_{1}\right\|_{p}\|g\|_{p}+C_{p}\left\|k_{2}\right\|_{p}\|g\|_{p} \leqq\left(1+C_{p}\right)\|k\|_{p}\|g\|_{p} .
$$

Thus the convolution operator $K$ with kernel $k$ is everywhere defined and bounded, in fact $\|K\| \leqq\left(1+C_{p}\right)\|k\|_{p}$. Next we show that $K \in$ strongly closed algebra generated by $\left\{T_{\lambda}\right\}_{\lambda \geqq 0}$.

For each positive integer $n$, set $k_{n}=\chi_{[0, n]} k$ and let $K_{n}$ be the convolution operator on $L^{p}(d \sigma)$ with kernel $k_{n}$. Then $\left\|K-K_{n}\right\| \leqq\left(1+C_{p}\right)\left\|k-k_{n}\right\|_{p} \rightarrow 0$ as $n \rightarrow \infty$. The lemma will follow at once if we can show that each $K_{n} \in$ strongly closed algebra generated by $\left\{T_{\lambda}\right\}_{\lambda \geqq 0}$.

Now $K_{n}$ can also be defined on $L^{p}(d \sigma)$ by the Bochner integral

$$
K_{n} g=\int_{0}^{n}\left(T_{t} g\right) k_{n}(t) d t
$$

The existence of the Bochner integral follows from the estimate

$$
\int_{0}^{n}\left\|T_{t} g\right\|_{p}\left|k_{n}(t)\right| d t \leqq n^{1 / q}\|k\|_{p}\|g\|_{p}
$$

Thus by Lemma 1 we have $K_{n} \in$ strongly closed algebra generated by $\left\{T_{\lambda}\right\}_{\lambda \geqq 0}$. This proves the lemma.

Theorem 4. Suppose $\sigma$ satisfies Property $\mathrm{P}$ and $k \in L^{p}(d \sigma)$ satisfies

$$
\text { closed span of }\left\{T_{\lambda} k\right\}_{\lambda \geqq 0}=L^{p}(d \sigma) \text {. }
$$

Let $K$ be the convolution operator on $L^{p}(d \sigma)$ with kernel $k$. Then the following are equivalent:

(i) $k$ is a cyclic vector for $K$,

(ii) strongly closed algebra generated by $K=$ strongly closed algebra generated by $\left\{T_{\lambda}\right\}_{\lambda \geqq 0}$,

(iii) $K$ and $\left\{T_{\lambda}\right\}_{\lambda \geqq 0}$ have the same collection of closed invariant subspaces.

The proof of Theorem 4 is essentially the same as the proof of Theorem 1 and its corollary, except we must use Lemma 5 in place of Lemma 1. Also we must use the norm estimate for convolution product given in Lemma 5. Hence we omit the proof of Theorem 4 .

Theorem 5. Suppose $\sigma$ satisfies Property $\mathrm{P}$ with $\beta(t)<1$ for all $t>0$. Suppose $k$ is in either $L^{p}(d \sigma)$ or $L^{1}\left(\left\|T_{t}\right\| d t\right)$. Then the convolution operator $K$ on $L^{p}(d \sigma)$ with kernel $k$ is Volterra. 
Proof. In the usual manner we can construct a sequence of convolution operators $\left\{K_{n}\right\}_{n=1}^{\infty}$ whose kernels $k_{n}$ are continuous functions on $(0, \infty)$ with compact support such that $K=\lim K_{n}$ in operator norm. If we can show each $K_{n}$ is Volterra, then it follows that $K$ must be Volterra.

Hence it suffices to assume that the kernel $k$ of $K$ is a continuous function on $(0, \infty)$ with compact support, say supp $k \subseteq[a, b]$ where $0<a<b<\infty$. For each positive integer $n$ set

$$
\begin{aligned}
& P_{n}=\text { the projection of } L^{p}(d \sigma) \text { onto } \chi_{[0, n]} L^{p}(d \sigma), \\
& Q_{n}=\text { the projection of } L^{p}(d \sigma) \text { onto } \chi_{[n, \infty)} L^{p}(d \sigma) .
\end{aligned}
$$

We intend to show that $K=\lim P_{n} K P_{n}$ in operator norm. Now each $P_{n} K P_{n}$ is essentially the convolution operator on $P_{n} L^{p}(d \sigma)$ with kernel $P_{n} k$. As noted earlier, such operators are Volterra. Thus it will follow that $K$ is Volterra once we establish that $K=\lim P_{n} K P_{n}$.

A short calculation using Property $P$ yields the estimates:

$$
\left\|T_{\lambda} Q_{n}\right\| \leqq \alpha(\lambda)^{1 / p} \beta(\lambda)^{n / p}, \quad\left\|Q_{n} T_{\lambda}\right\| \leqq \alpha(\lambda)^{1 / p} \beta(\lambda)^{(n-\lambda) / p}, \quad n \geqq \lambda .
$$

Thus by the bounded convergence theorem we get

$$
\begin{aligned}
\left\|K Q_{n}\right\| & \leqq \int_{a}^{b}\left\|T_{t} Q_{n}\right\||k(t)| d t \\
& \leqq \int_{a}^{b} \alpha(t)^{1 / p} \beta(t)^{n / o}|k(t)| d t \rightarrow 0 \quad \text { as } n \rightarrow \infty, \\
\left\|Q_{n} K\right\| & \leqq \int_{a}^{b}\left\|Q_{n} T_{t}\right\||k(t)| d t \\
& \leqq \int_{a}^{b} \alpha(t)^{1 / p} \beta(t)^{(n-b) / p}|k(t)| d t \rightarrow 0 \quad \text { as } n \rightarrow \infty .
\end{aligned}
$$

Now it follows in a straightforward manner using the triangle inequality that $K=\lim P_{n} K P_{n}$. This completes the proof of the theorem.

Finally we offer a method of constructing measures $\sigma$ satisfying Property $P$. Suppose $\sigma$ is a measure on $[0, \infty)$ satisfying the following property:

Property Q. $\sigma$ is a measure on $[0, \infty)$ of the form $d \sigma=\alpha d x$ where

$$
\alpha(x)=\exp \left(\int_{0}^{x} \log \beta(t) d t\right)
$$

where $\beta$ is a nonincreasing function on $(0, \infty)$ satisfying

(i) $0 \leqq \beta(t)<1$ for all $t>0$,

(ii) $\beta$ is submultiplicative,

(iii) $C_{p}=\left[\int_{0}^{\infty} \beta(t)^{q / p} d t\right]^{1 / q}<\infty$.

It is a straightforward exercise to show that Property $\mathbf{Q}$ implies Property $\mathbf{P}$. An example of a function $\alpha$ satisfying the conditions in Property $Q$ is $\alpha(x)=$ 
$\exp \left(-a x^{s}\right)$ where $a>0$ and $s \geqq 2$. The corresponding function $\beta$ is given by $\beta(x)$ $=\exp \left(-a s x^{s-1}\right)$.

Conjecture. If $\sigma$ is a measure on $[0, \infty)$ satisfying Property $Q$, then the lattice of closed right translation invariant subspaces of $L^{p}(d \sigma)$ forms a chain under set inclusion.

5. The Lebesgue spaces $L^{p}(0, \infty)$ and $L^{p}\left(e^{-x} d x\right)$. In this section we study convolution operators on the Lebesgue spaces $L^{p}(0, \infty)$ and $L^{p}\left(e^{-x} d x\right)$. Both of these spaces are examples of $L^{p}(d \sigma)$ spaces where $\sigma$ is a measure on $[0, \infty)$ of the form $d \sigma=\alpha d x$ where $\alpha \geqq 0$ is a nonincreasing submultiplicative function on $(0, \infty)$. The study of convolution operators on $L^{p}(0, \infty)$ is closely related to the study of convolution operators on $L^{p}\left(e^{-x} d x\right)$.

Consider the isometry $U: L^{p}(0, \infty) \rightarrow L^{p}\left(e^{-x} d x\right)$ defined by $(U f) x=e^{x / p} f(x)$. It is easily checked that $U$ is an isometry of $L^{p}(0, \infty)$ onto $L^{p}\left(e^{-x} d x\right)$ and that $U^{-1}: L^{p}\left(e^{-x} d x\right) \rightarrow L^{p}(0, \infty)$ is given by $\left(U^{-1} g\right) x=e^{-x / p} g(x)$.

Suppose $K$ is a convolution operator on $L^{p}(0, \infty)$ with kernel $k$. A short calculation shows that $K_{0}=U K U^{-1}$ is a convolution operator on $L^{p}\left(e^{-x} d x\right)$ with kernel $k_{0}=U k$. Thus $U$ provides an isometric equivalence between the convolution operators on $L^{p}(0, \infty)$ and those on $L^{p}\left(e^{-x} d x\right)$.

Likewise one can show that $U$ provides an isometric equivalence between the right translation operators $\left\{T_{\lambda}\right\}_{\lambda \geqq 0}$ on $L^{p}(0, \infty)$ and the operators $\left\{e^{\lambda / p} T_{\lambda}^{\prime}\right\}_{\lambda \geqq 0}$ on $L^{p}\left(e^{-x} d x\right)$ where $\left\{T_{\lambda}^{\prime}\right\}_{\lambda \geqq 0}$ denotes the collection of right translation operators on $L^{p}\left(e^{-x} d x\right)$. An immediate consequence is that $U$ maps the lattice of closed right translation invariant subspaces of $L^{p}(0, \infty)$ one-to-one onto the lattice of closed right translation invariant subspaces of $L^{p}\left(e^{-x} d x\right)$.

The remainder of this section will be devoted to a study of convolution operators on $L^{p}(0, \infty)$ as any results obtained will be applicable to $L^{p}\left(e^{-x} d x\right)$ as well.

Next we show that no convolution operator on either $L^{1}(0, \infty)$ or $L^{2}(0, \infty)$ is unicellular if its kernel is in $L^{1}(0, \infty)$. By Lemma 1 all we need show is that the lattice of closed right translation invariant subspaces of either space does not form a chain under set inclusion.

The structure of the lattice of closed right translation invariant subspaces of $L^{2}(0, \infty)$ is well known and does not form a chain under set inclusion (see Hoffman [2]). This lattice is identical to the lattice of closed invariant subspaces of the Laguerre shift $S$ on $L^{2}(0, \infty)$ defined by $S: \phi_{n} \rightarrow \phi_{n+1}$ where $\left\{\phi_{n}\right\}_{n=0}^{\infty}$ is the orthonormal basis of Laguerre functions.

Next we show that the lattice of closed right translation invariant subspaces of $L^{1}(0, \infty)$ does not form a chain under set inclusion. Suppose $g \in L^{1}(0, \infty)$. In order that

$$
\text { closed } \operatorname{span}\left\{T_{\lambda} g\right\}_{\lambda \geqq 0}=L^{1}(0, \infty)
$$

it is necessary that $\mathscr{F} g$ never vanish on $(-\infty, \infty)$ where $\mathscr{F} g$ denotes the Fourier transform of $g$. This result follows directly from the methods used in a proof of a 
theorem of Wiener [4]. Taking $g=\chi_{[0,1]}$ we get $(\mathscr{F} g) x=\left(e^{i x}-1\right) /\left(i x(2 \pi)^{1 / 2}\right)$. Clearly $\mathscr{F} g$ vanishes at $x=2 \pi n$ for all integers $n \neq 0$. Thus $M=$ closed span $\left\{T_{\lambda} g\right\}_{\lambda} \geqq 0$ $\neq L^{1}(0, \infty)$. But $M \neq L^{1}(a, \infty)$ for any $a>0$ since $l_{g}=0$. Thus $M \neq L^{1}(a, \infty)$ for any $a \geqq 0$, proving that the lattice of closed right translation invariant subspaces of $L^{1}(0, \infty)$ does not form a chain under set inclusion.

Finally we remark that there are no nonzero compact convolution operators on $L^{p}(0, \infty)$. See, for example, Kreìn [3].

\section{REFERENCES}

1. S. Agmon, Sur un problème de translations, C. R. Acad. Sci. Paris 229 (1949), 540-542. MR 11, 101.

2. K. Hoffman, Banach spaces of analytic functions, Prentice-Hall Series in Modern Analysis, Prentice-Hall, Englewood Cliffs, N. J., 1962. MR 24 \#A2844.

3. M. G. Kreìn, Integral equations on a half-line with kernel depending upon the difference of the arguments, Uspehi Mat. Nauk 13 (1958), no. 5 (83), 3-120; English transl., Amer. Math. Soc. Transl. (2) 22 (1962), 163-288. MR 21 \#1507.

4. N. Wiener, The Fourier integral and certain of its applications, Cambridge Univ. Press, Cambridge, 1933; reprint, Dover, New York, 1959. MR 20 \#6634.

5. K. Yosida, Functional analysis, Die Grundlehren der math. Wissenschaften, Band 123, Academic Press, New York; Springer-Verlag, Berlin, 1965. MR 31 \#5054.

Department of Mathematics, University of Wyoming, LaRamie, Wyoming 82070 